\title{
Retraction Note to: Comparison of hepatocellular carcinoma (HCC), cholangiocarcinoma (CC), and combined HCC-CC (CHC) with each other based on microarray dataset
}

\author{
Lishan Wang
}

Published online: 17 August 2015

(C) International Society of Oncology and BioMarkers (ISOBM) 2015

Retraction to: Tumor Biol. (2013) 34:1679-1684

DOI 10.1007/s13277-013-0702-6

The Publisher and Editor retract this article in accordance with the recommendations of the Committee on Publication Ethics (COPE). After a thorough investigation we have strong reason to believe that the peer review process was compromised.

Update posted 26th June 2020

Springer Nature has been informed by Shanghai Jiao Tong University that it has conducted an investigation that found that ( i ) Lishan Wang is the sole author responsible for this article and the actual corresponding author and (ii) none of the other listed co-authors were aware of this submission or any related correspondence and were included by Lishan Wang without notification. 